\title{
APLICACIÓN DEL MÉTODO BAYES EMPÍRICO EN ANÁLISIS DE SEGURIDAD VIAL: EL CASO DE LA RUTA 32 EN COSTA RICA
}

\author{
Esteban Alberto Castro Estrada \\ Jonathan Agüero Valverde
}

\begin{abstract}
Resumen
Existe una problemática evidente en la frecuencia y severidad de choques en la Ruta Nacional 32 en Costa Rica. Se realiza un análisis de tramos de concentración de choques, debido a que es una de las carreteras más importantes del país, y que además representa prácticamente la única vía directa de comunicación entre la Gran Área Metropolitana y la Vertiente Atlántica. Para realizar este análisis se utilizó el Método de exceso de frecuencia de choques esperado con el ajuste de Bayes empírico. Este método toma en cuenta tres aspectos importantes que lo hacen más preciso que otros: la disponibilidad de datos, el sesgo de regresión a la media y cómo se establece el umbral de desempeño. Una vez procesadas las bases de datos de choques y el inventario vial de la ruta se procedió a utilizar el método de Bayes empírico, con el cual se determinó el exceso de frecuencia de choques viales con respecto a la cantidad que se considera como esperable según la función de desempeño. Los resultados muestran que el TPD, el número de carriles y la densidad de accesos están positivamente relacionados con la frecuencia de choques. Además, los tramos de concentración de choques se ubican principalmente en la parte plana de la ruta a la entrada de los principales poblados de la región.
\end{abstract}

Palabras Clave: Carretera Braulio Carillo (Costa Rica), accidentes de tránsito-procesamiento de datos-Costa Rica, accidentes de tránsito-identificación-Costa Rica, percepción de riesgo, accidentes de tránsito-identificación-Costa Rica.

\begin{abstract}
There is an evident problem of crash frequency and severity on the National Route 32 in Costa Rica. Since it is one of the most important roads in the country, and virtually the only direct route between the Greater Metropolitan Area and the Atlantic Coast, the selection of "sites with promise" is performed. The method of excess expected crash frequency with empirical Bayes adjustment was used for this analysis. This method takes into account three important aspects that make it more accurate than others: it uses more data, it accounts for regression to the mean bias, and a performance threshold is set. After processing the crash database and the roadway inventory the empirical Bayes method was applied, which determined the excess on the expected number of crashes with respect to the safety performance function. The results showed that ADT, number of lanes, and access density are positively correlated with crash frequency. Furthermore, the "sites with promise" were identified mainly in the flat part of the road near the main population centes in the region.
\end{abstract}

Keywords: Safety Performance Function, empirical Bayes, sites with promise, Route 32, excess expected crash frequency

Recibido: 2 de diciembre 2014 Aprobado: 2014

\section{INTRODUCCIÓN}

Según la Organización Mundial de la Salud (World Health Organization, 2013), aproximadamente 1,24 millones de personas mueren cada año en carretera alrededor del mundo, siendo los choques viales la octava causa de muerte a nivel global. En Costa Rica la situación es similar; los accidentes del transporte fueron la octava causa de muerte en el 2012, produciendo 661 decesos (Centro Centroamericano de Población (CCP), 2014). Más aún, en Costa 
Rica los choques viales fueron la primera causa de muerte para personas entre 0 y 44 años de edad en el 2012 (CCP, 2014). Este problema es particularmente agudo por la población en riesgo: por un lado ataca a la población más vulnerable, menores de edad. y por otro diezma a una parte significativa de la población económicamente activa. En términos de años de vida potenciales perdidos los accidentes del transporte fueron la tercera causa de muerte en Costa Rica en el 2012, solo superados por el cáncer y enfermedades cardiovasculares (CCP, 2014).

Más allá de la seriedad de las consecuencias sociales y económicas de los choques viales para el país, la complejidad del problema complica la búsqueda de soluciones. Los choques viales son el resultado de una falla en el complejo sistema de interacciones entre el usuario, el vehículo y el medioambiente. Este último ámbito es donde la ingeniería de transportes puede dar su mayor contribución, particularmente en la interacción entre el usuario y la carretera, según las últimas tendencias en el ramo. Dado que la mayoría de los choques viales ocurren por errores humanos (Shinar, 2007), las medidas de ingeniería en seguridad vial actuales deben buscar la mejor interacción entre el usuario, la carretera y su entorno.

Una de las tareas fundamentales para la mejora de la seguridad vial es la identificación de tramos de concentración de choques. Este proceso busca la identificación y ordenamiento de sitios (segmentos de carretera o intersecciones) de una red de transporte, del más probable al menos probable en presentar una reducción en la frecuencia de choques con la implementación de una medida de mejora de seguridad vial (ASSHTO, 2010). Existen varias medidas de desempeño para ordenar los sitios, entre los cuales destaca el valor esperado de exceso de frecuencia de choques con corrección de Bayes empírico.

El método de valor esperado de exceso de frecuencia de choques con corrección de Bayes empírico es ampliamente reconocido como uno de los métodos más efectivos para la clasificación de sitios de concentración de choques, debido a que puede corregir por regresión a la media y puede determinar si un sitio presenta un exceso de choques estadísticamente significativo, con respecto al valor esperado para sitios similares (ASSHTO, 2010; World Road Association, 2003). A pesar de lo anteriormente expuesto, se conocen muy pocas aplicaciones del método en el medio latinoamericano (Rizzi, 2001; da Costa, Barbosa, 2014).

La Ruta 32 es un corredor vial de gran importancia en Costa Rica, pues permite en trasiego de personas y mercancías desde el principal puerto del país en el Caribe costarricense a la capital. Pese a su importancia, la Ruta 32 se considera peligrosa ya que posee varias características que la alejan de las condiciones ideales. De acuerdo con estudios realizados por la Unidad de Evaluación de la Red Vial Nacional del LANAMME-UCR (2011):

- Una gran parte del terreno es ondulado o montañoso

- Presenta varios tramos con un alineamiento horizontal sinuoso

- Llueve más del 50\% de los días del año

- La precipitación de la carretera es mayor a $3250 \mathrm{~mm} / \mathrm{año}$

- Las condiciones de la demarcación eran malas o inexistentes al momento del estudio

- El agarre superficial en su mayoría es bastante deficiente

El objetivo de este estudio es determinar los tramos de concentración de choques de la Ruta 32 utilizando el método de valor esperado de exceso de frecuencia de choques con corrección de Bayes empírico. Este trabajo está organizado de la siguiente manera: se presenta la metodología utilizada, luego se realiza una breve descripción de los datos utilizados para dar paso a la discusión de resultados, finalmente se presentan conclusiones y recomendaciones.

\section{METODOLOGÍA}

El método de Bayes empírico busca realizar una estimación del número de choques esperado en un sitio usando tanto los datos de frecuencia de choques observados en el sitio, así como la evidencia proveniente de otros sitios con características similares. Para tal efecto se utiliza la función de desempeño de seguridad vial. Para la estimación de la función de desempeño de seguridad vial se deben realizar los análisis estadísticos que se detallan a continuación. 
Si se considera cada viaje como un evento de Bernouilli con una probabilidad $p$ (muy baja) de terminar en un choque y se agregan dichos viajes sobre un tiempo determinado en una sección de carretera o intersección, el número de choques observados tendrá una distribución de Poisson. Ahora bien, se conoce que los choques viales presentan más dispersión de la que una distribución de Poisson puede explicar, dado que la varianza en dicha distribución está restringida a ser igual que el promedio (Shankar, Mannering \& Barfield, 1995). Por lo anterior, es común que se utilice la distribución binomial negativa que se basa en la distribución de Poisson pero permite que la varianza sea mayor que el promedio (Agüero-Valverde y Jovanis, 2006). Los detalles estadísticos de la estimación de la distribución binomial negativa escapan al alcance de este trabajo, los lectores interesados en más detalles pueden consultar Agüero-Valverde (2005).

La función de desempeño es estimada usando un modelo generalizado lineal con una distribución de probabilidad binomial negativa. La tasa de choques de la distribución es estimada como una función de la exposición y otras variables características de los segmentos como se muestra en la Ecuación 1:

$\ln ($ choques $)=\beta_{0}+\beta_{1} \cdot \ln ($ TPDA $)+\beta_{2} \cdot$ num

carriles $+\beta_{2} \cdot$ dens accesos $+\ln ($ Long $)$

Donde $\beta_{0}$ es el término correspondiente al intercepto, $\beta_{l}$ es el coeficiente extraído del modelo y se incluyen las variables de TPDA y longitud del segmento como medida de la exposición. Además, $\beta_{2}$ es el coeficiente correspondiente al número de carriles y $\beta_{3}$ es el coeficiente correspondiente a la densidad de accesos.

Una vez estimados los coeficientes, el número predicho de choques es estimado por medio de la función de desempeño de seguridad, la cual es:

$N_{\text {pred }}=e^{\beta_{0}} \cdot T P D A^{\beta_{1}} \cdot e^{\left(\beta_{2} \cdot \text { núm carriles }\right)} \cdot e^{\left(\beta_{3} \cdot \text { dens accesos }\right)}$ - Longitud

Seguidamente, se calcula lo que se conoce como ajuste por peso, que se refiere a la confiabilidad de la función de desempeño de seguridad que se está utilizando. Si los parámetros de sobredispersión son bajos, lo cual indica una mayor confiabilidad, entonces se tendrá un ajuste por peso más grande, que a su vez indica que la función de desempeño es más confiable. El peso se calcula de la siguiente manera:

$w=1 /\left(1+k\left(N_{\text {pred }}\right)\right)$

En donde $k$ es el parámetro de sobredispersión de la función de desempeño de seguridad. En este caso en específico, w se conoce como el peso de Bayes empírico.

Finalmente, la frecuencia de choques esperados total se calcula por medio de la siguiente ecuación:

$N_{\text {exp }}=w \cdot N_{\text {pred }}+(1-w) \cdot N_{o b s}$

En la cual la variable de $N_{o b s}$ corresponde al número de choques observados, es decir, los choques que se tienen reportados por parte de los oficiales de tránsito y que fueron recopilados anteriormente.

El paso siguiente consiste en calcular el exceso de frecuencia de choques esperado, que corresponde a la diferencia entre los estimados predichos y los estimados con el ajuste de Bayes empírico:

$\Delta=N_{\text {exp }}-N_{\text {pred }}$

Dicho exceso permite identificar cuáles son los tramos que tienen una mayor frecuencia de choques esperada que los estimados predichos, y se espera van a responder mejor a las medidas de mitigación propuestas, puesto que poseen un exceso importante que puede disminuirse. La Figura 1 muestra de forma gráfica las magnitudes señaladas en las Ecuaciones 1 a 5 .

La función de desempeño de seguridad vial se presenta en valor predicho de choques para segmentos con características similares al segmento en estudio. Luego con el peso de Bayes empírico se realiza la corrección por sesgo de regresión a la media, por lo que el valor esperado $\left(N_{\text {exp }}\right)$ es un valor intermedio entre la frecuencia observada $\left(N_{o b s}\right)$ y la frecuencia predicha por la función de desempeño $\left(N_{\text {pred }}\right)$.

El concepto de choques equivalentes se utiliza para equiparar o comparar las distintas severidades de choque (Oh, Washington \& Lee, 2010). Como no existen análisis de los costos de choques para cada tipo de gravedad en Costa Rica, no se pueden establecer los 


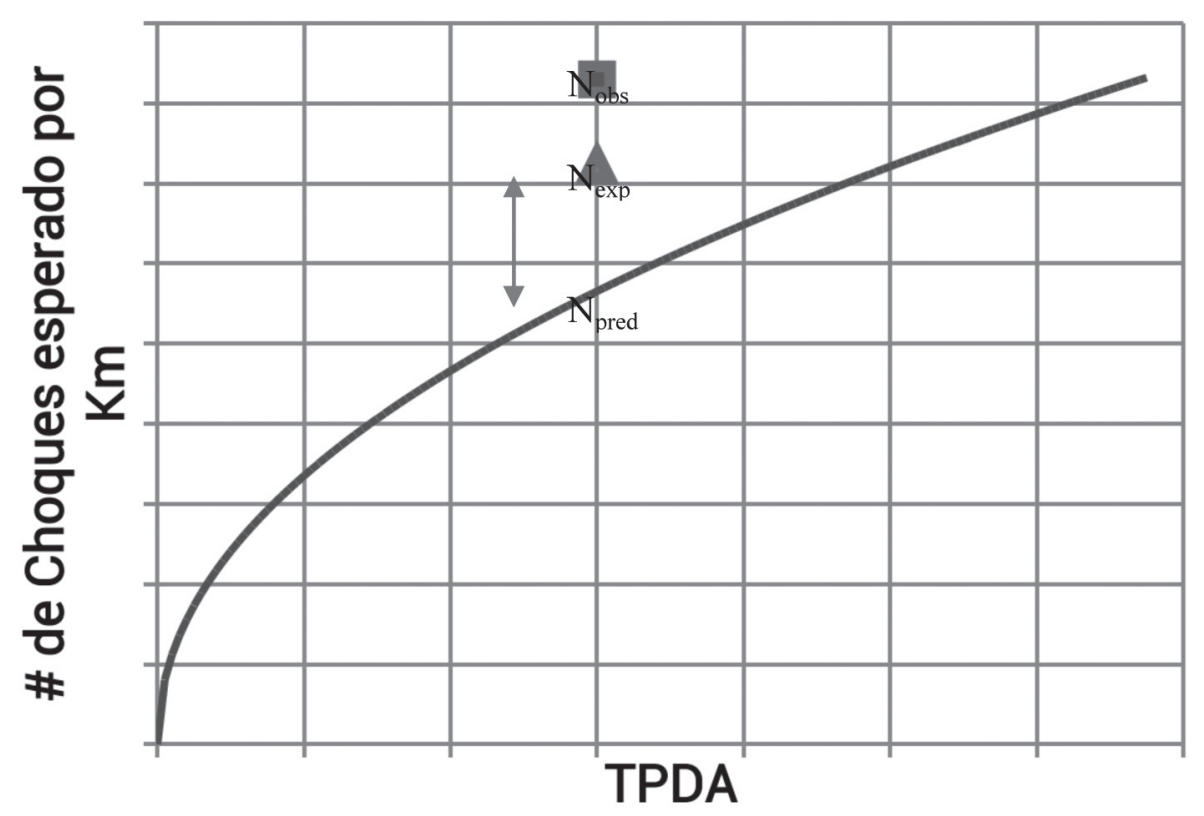

Figura 1. Función de Desempeño de Seguridad Vial

factores de equivalencia de choques para el país, por lo cual se recurrió al análisis de factores de equivalencia de choques en otros países. Dichos estudios muestran valores muy discordantes, por ejemplo un estudio de costos de choques en Gran Bretaña en el 2011 (Department of Transport, 2012) reveló factores de equivalencia a solo daños materiales de 926, 107 y 11 para choques fatales, con heridos graves y con heridos leves respectivamente. Por otro lado, las estadísticas de costos en Pensylvania, EUA (Pennsylvania Department of Transportation, 2012) para el 2011 muestran factores de equivalencia de 2155, 471,31 y 3 para choques fatales, con heridos graves, con heridos moderados y con heridos leves respectivamente. La perspectiva de otro país desarrollado se muestra en Nueva Zelanda donde los datos de costos de choques para el 2012 muestran factores de equivalencia a solo daños materiales de 1612,168 y 10 para choques fatales, con heridos graves y con heridos leves respectivamente (Ministry of Transport, 2013).

Las estadísticas en términos de los costos de los choques viales para países latinoamericanos son escasas. El Ministerio de Transporte de Chile publicó en su manual de Tratamiento de Puntos Negros equivalencias de choques de 34 y 11 para choques fatales y con heridos graves respectivamente, pero las equivalencias corresponden a choques con heridos leves, no a choques con solo daños materiales (CONASET, 2008). Peniche (2007) reportó para la Ciudad de Campeche, México, costos de choques que significan una equivalencia a choques de solo daños materiales de 40, 12 y 3 para choques fatales, con heridos graves y con heridos leves respectivamente. Dado el panorama anteriormente expuesto, se seleccionaron para este estudio los factores de equivalencia a solo daños materiales a partir de los datos de costos de choques estimados para Campeche.

Una vez seleccionados los sitios de concentración de choques de la Ruta 32 con el procedimiento anteriormente descrito, se procedió a realizar un estudio de seguridad vial de cada uno de los sitios. Dicho estudio incluyó tanto un análisis de la información de las boletas de choques disponible, como un análisis en campo de las características de los segmentos de carretera en cuestión. El resultado final del análisis fue la propuesta de mejoras para la reducción de la frecuencia y gravedad de choques viales en dichos tramos. Los lectores interesados en mayores detalles metodológicos así como el análisis detallado de cada tramo de concentración de choques pueden consultar la tesis del Ing. Castro (2013). 


\section{DESCRIPCIÓN DE LOS DATOS}

\subsection{Datos de los choques}

Los datos de choques corresponden a las años 2008 a 2011 y fueron suministrados por el Consejo de Seguridad Vial del Ministerio de obras Públicas y Transportes de Costa Rica (MOPT). Para el análisis de choques equivalentes a daños materiales, los choques fueron agregados según su gravedad. La Figura 2 muestra la cantidad de choques en toda la ruta según su gravedad. Además, como se mencionó anteriormente, la información detallada de cada choque se utilizó para realizar el diagnóstico de los sitios de concentración de choques.

\subsection{Datos de los segmentos}

La información base de red vial, en este caso de la Ruta 32, proviene del Departamento de Planificación Sectorial también del MOPT. Dicha información fue complementada con trabajo de campo para realizar una verificación de los datos y completar la base con variables tales como el número de accesos que no se incluía en el inventario de la red vial nacional que suministró el MOPT. La ruta fue dividida en 98 segmentos homogéneos basados en sus características geométricas, tales como número de carriles, alineamiento horizontal, alineamiento vertical y uso del suelo circundante entre otros. $\mathrm{La}$ Tabla 1 presenta las estadísticas descriptivas por segmento para los datos de frecuencia de choques.

\section{RESULTADOS}

\subsection{Funciones de desempeño de seguridad}

Las funciones de desempeño de seguridad para la Ruta 32 fueron estimadas tanto para el total de choques como para el número equivalente a solo daños materiales, que fue determinado según se explicó en la sección de metodología. Las variables incluidas en los modelos finales son:

- TPDA

- Longitud (medida de exposición, coeficiente igual a 1)

- Número de carriles

- Densidad de accesos

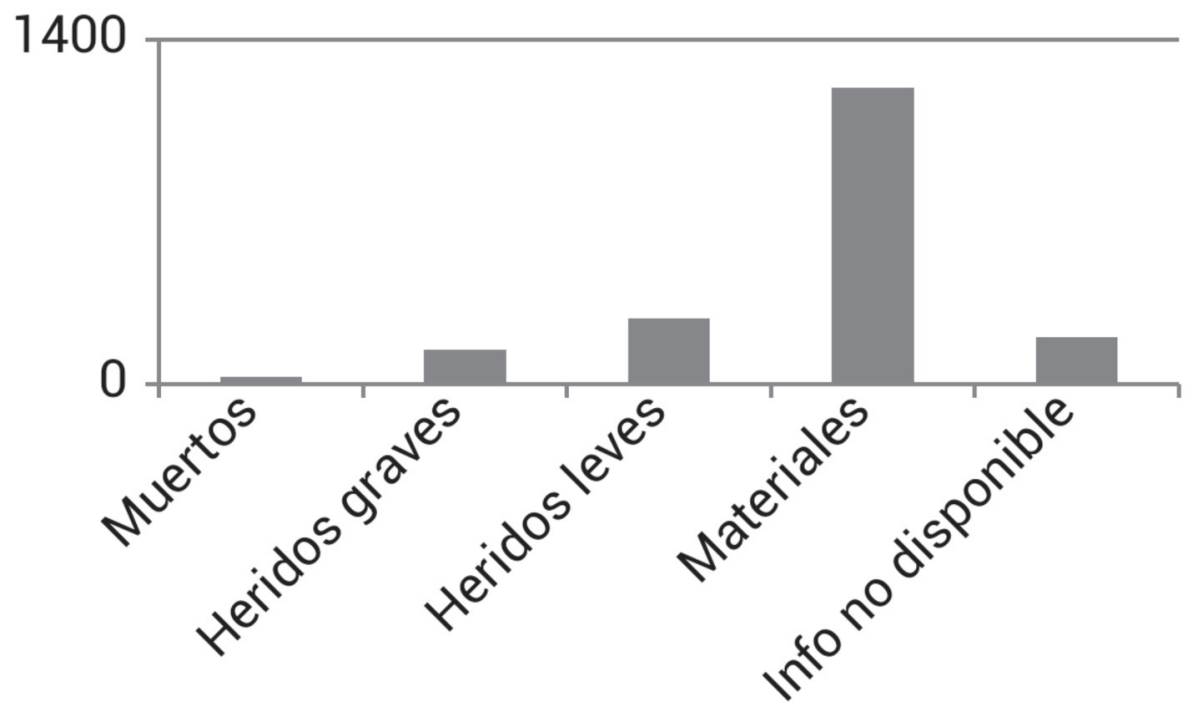

Figura 2. Frecuencia de Choques según Gravedad. 
Tabla 1. Estadísticas descriptivas por segmento y gravedad de choque.

\begin{tabular}{lccccccc}
\hline & Mínimo & 1er Cuartil & Mediana & Promedio & 3er Cuartil & Máximo & Desv. Est. \\
\hline Mortales & 0 & 0 & 0 & 0.24 & 0 & 2 & 0.52 \\
Heridos graves & 0 & 0 & 0 & 1.13 & 1 & 10 & 1.85 \\
Heridos leves & 0 & 0 & 1 & 2.21 & 3 & 19 & 3.2 \\
Sólo daños materiales & 0 & 1.25 & 4.5 & 9.8 & 11 & 134 & 16.7 \\
Totales & 0 & 3 & 7 & 14.88 & 14.25 & 171 & 22.73 \\
\hline
\end{tabular}

Un gran número de otras variables, tales como curvatura de la sección, tipo y condición de la calzada y ancho de carril y espaldón fueron analizadas en los modelos preliminares, sin embargo, estas variables no resultaron significativas, por lo que no se incluyeron en los modelos finales. Además, se estimaron modelos para solo choques fatales y solo choques con heridos pero dichos modelos no resultaron estadísticamente significativos, dado el bajo número de secciones en el modelo (98) y el bajo valor esperado para estos niveles de gravedad $(0,24$ choques fatales y 3,34 choques con heridos por segmento) comparado con el de total de choques (14.88 choques por segmento) (Lord, Washington e Ivan, 2005).

La Tabla 2 muestra los resultados para el modelo de choques totales. Tanto la variable del flujo vehicular como la de densidad de accesos advierten que entre mayor sea la magnitud de dichas variables, mayor será el riesgo de choques, lo cual es la condición esperada según estudios previos (Jovanis and Chang, 1986; Miaou, 1994; Shankar et al, 1995; Harwood, Council, Hauer, Hughes \& Vogt, 2000).

De la Tabla 2 se observa que el coeficiente para TPDA es además significativamente menor a 1, lo que indica que la relación entre la frecuencia esperada de choques y el TPDA no es lineal. Más aún, un coeficiente para el TPDA menor a 1, significa que la relación entre el número de choques y el TPDA es creciente pero a un ritmo decreciente.
Por otro lado, los resultados también revelan que si el tramo tiene tres o más carriles, el riesgo de choques también va a aumentar. Esto no coincide con investigaciones anteriores (Park, Fitzpatrick $\&$ Brewerl, 2012), pero hay que tener en cuenta que los tramos en los que existen más de dos carriles tienen condiciones especiales, como lo son carriles de ascenso o incluso poblaciones con gran cantidad de accesos y tráfico vehicular, por lo que es más probable la incidencia de choques. Esto se ve reflejado en la significancia de las variables.

Como se mencionó anteriormente, para el caso de choques equivalentes a solo daños materiales se utilizaron los pesos de 40, 12 y 3 para choques fatales, con heridos graves y con heridos leves respectivamente. La Tabla 3 muestra los resultados del modelo para choques equivalentes a solo daños materiales.

Los resultados del modelo de choques equivalentes son consistentes con el modelo de choques totales aunque la variable de número de carriles no es estadísticamente significativa, y en coeficiente para el TPDA es muy poco significativo (la probabilidad de rechazo es de un $13 \%$ ). Dado que se tiene un tamaño de muestra relativamente reducido de 98 segmentos, estos resultados no son sorprendentes.

Aquí, la variable más significativa es la densidad de accesos, lo cual concuerda con estudios anteriores ya que entre más intersecciones y entradas y salidas de vehículos hayan, mayores son el riesgo y frecuencia de los choques. Cabe mencionar que la cantidad de accesos puede estar 
CASTRO Y AGÜERO: Aplicación del método Bayes empírico...

Tabla 2. Resultado del modelo binomial negativo para choques totales.

\begin{tabular}{lccccc}
\hline & Coeficiente & $\begin{array}{c}\text { Error } \\
\text { estándar }\end{array}$ & Valor de $\mathbf{z}$ & $\operatorname{Pr}(>\mid \mathbf{z} \mathbf{l})$ & Significancia \\
\hline Constante & -10.41475 & 1.57885 & -6.596 & $4.21 \mathrm{E}-11$ & $* * *$ \\
$\ln (\mathrm{TPDA})$ & 0.47623 & 0.15079 & 3.158 & 0.00159 & $* *$ \\
más de 2 carriles & 0.24783 & 0.14224 & 1.742 & 0.08145 & \\
& 0.08198 & 0.01076 & 7.621 & $2.51 \mathrm{E}-14$ & $* * *$ \\
\hline
\end{tabular}

Nota: Códigos significancia: '***' $0.001 \quad{ }^{\prime * * 1} 0.01 \quad$ '*' $0.05 \quad$ '.'0.1. Desviación nula: 186.69 en 97 grados de libertad. Desviación residual: 107.64 en 94 grados de libertad AIC: 598.2.2 x log-likelihood: -588.202. $\theta(1 / \mathrm{k}): 3.905$. Error estándar: 0.856 .

Tabla 3. Resultado del modelo binomial negativo para choques equivalentes.

\begin{tabular}{lccccc}
\hline & Coeficiente & $\begin{array}{c}\text { Error } \\
\text { estándar }\end{array}$ & Valor de $\mathbf{z}$ & $\operatorname{Pr}(>\mathbf{z l})$ & Significancia \\
\hline Constante & -8.24583 & 2.6716 & -3.086 & $2.03 \mathrm{E}-03$ & $* *$ \\
$\log (\mathrm{TPDA})$ & 0.38063 & 0.25565 & 1.489 & 0.136513 & \\
más de 2 carriles & 0.18895 & 0.22338 & 0.846 & 0.398 & $*$ ** \\
Densidad de accesos & 0.06615 & 0.01813 & 3.649 & 0.000263 & \\
\hline
\end{tabular}

Nota: Códigos significancia: '***' $0.001 \quad$ '**' $0.01 \quad$ '*' $0.05 \quad$ '.' 0.1 Desviación nula: 135.81 en 97 grados de libertad. Desviación residual: 114.28 en 94 grados de libertad. AIC: 838.41. 2 x log-likelihood: -828.415. $\theta(1 / \mathrm{k}): 1.068$. Error estándar: 0.16

relacionada con el tráfico local. Dicho tráfico local no se ve reflejado en las estaciones de recuento vehicular utilizadas para realizar el inventario de carreteras puesto que el interés es en los flujos regionales no locales.

\subsection{Método de exceso de frecuencia de choques esperado con ajuste de Bayes empírico}

La Tabla 4 muestra los veinte tramos que presentan mayor exceso de frecuencia de choques esperado $(\Delta)$ para el total de choques. Además, la tabla presenta el exceso de choques equivalentes a solo daños materiales para los mismos segmentos así como la clasificación según esa variable. La Figura 3 muestra el mapa de los segmentos estudiados clasificados según el exceso de choques totales.

Un patrón que se hace evidente en el mapa de la Figura 3, es que los segmentos con mayor exceso por total de choques se presentan principalmente en las cercanías de las ciudades más importantes alrededor de la Ruta 32. Así por ejemplo, el segmento con mayor exceso se 
Tabla 4. Exceso de frecuencia de choques esperado $(\Delta)$ para el total de choques y para choques equivalentes a solo daños materiales.

\begin{tabular}{|c|c|c|c|c|c|c|c|c|c|c|}
\hline \multirow{2}{*}{ Tramo } & \multicolumn{5}{|l|}{ Total } & \multicolumn{5}{|c|}{ Equivalentes a solo daños materiales } \\
\hline & orden & $\mathbf{N}_{\text {pred }}$ & $\mathbf{N}_{\text {obs }}$ & $\mathbf{N}_{\text {exp }}$ & $\Delta$ & orden & $\mathbf{N}_{\text {pred }}$ & $\mathbf{N}_{\text {obs }}$ & $\mathbf{N}_{\text {exp }}$ & $\Delta$ \\
\hline 97 & 1 & 92.2 & 171 & 167.8 & 75.6 & 1 & 211.4 & 391 & 390.1 & 178.7 \\
\hline 96 & 2 & 16.5 & 49 & 42.8 & 26.3 & 4 & 43.3 & 123 & 121.1 & 77.8 \\
\hline 55 & 3 & 52.5 & 78 & 76.2 & 23.7 & 17 & 139.7 & 170 & 169.8 & 30.1 \\
\hline 25 & 4 & 16.4 & 40 & 35.5 & 19.0 & 6 & 50.7 & 122 & 120.5 & 69.8 \\
\hline 15 & 5 & 13.6 & 31 & 27.1 & 13.5 & 10 & 40.9 & 96 & 94.6 & 53.7 \\
\hline 59 & 6 & 17.8 & 33 & 30.3 & 12.5 & 8 & 55.9 & 113 & 111.9 & 56.0 \\
\hline 61 & 7 & 25.9 & 38 & 36.4 & 10.5 & 2 & 68.7 & 157 & 155.6 & 87.0 \\
\hline 5 & 8 & 17.4 & 30 & 27.7 & 10.3 & 67 & 47.7 & 38 & 38.2 & -9.5 \\
\hline 1 & 9 & 17.2 & 28 & 26.0 & 8.8 & 78 & 43.8 & 27 & 27.4 & -16.4 \\
\hline 63 & 10 & 33.7 & 43 & 42.0 & 8.3 & 75 & 100.0 & 86 & 86.1 & -13.9 \\
\hline 95 & 11 & 24.8 & 34 & 32.7 & 8.0 & 19 & 68.2 & 90 & 89.7 & 21.4 \\
\hline 67 & 12 & 17.7 & 27 & 25.3 & 7.6 & 11 & 42.2 & 91 & 89.8 & 47.5 \\
\hline 85 & 13 & 30.5 & 37 & 36.3 & 5.8 & 3 & 96.7 & 183 & 182.1 & 85.4 \\
\hline 43 & 14 & 21.2 & 28 & 26.9 & 5.7 & 87 & 51.8 & 24 & 24.6 & -27.3 \\
\hline 70 & 15 & 13.2 & 20 & 18.5 & 5.2 & 22 & 38.3 & 50 & 49.7 & 11.4 \\
\hline 72 & 16 & 35.1 & 40 & 39.5 & 4.4 & 5 & 104.3 & 180 & 179.2 & 75.0 \\
\hline 33 & 17 & 7.3 & 14 & 11.6 & 4.4 & 14 & 23.5 & 69 & 67.0 & 43.5 \\
\hline 90 & 18 & 5.8 & 12 & 9.5 & 3.7 & 9 & 17.8 & 75 & 71.8 & 54.0 \\
\hline 3 & 19 & 3.0 & 11 & 6.4 & 3.5 & 12 & 8.7 & 60 & 54.4 & 45.7 \\
\hline 38 & 20 & 3.7 & 9 & 6.3 & 2.6 & 13 & 10.8 & 61 & 56.5 & 45.7 \\
\hline
\end{tabular}


encuentra a la entrada de la Ciudad de Limón, la más importante en la ruta luego de la capital, San José (segmento de color rojo). Otros segmentos con excesos significativos de choques totales se encuentran en las cercanías de otros poblados importantes como Matina, Guápiles, y Siquirres. Es importante recalcar que aun cuando el modelo controla por número de accesos y volumen vehicular, los segmentos con concentración de choques alrededor de poblados donde existen una mezcla de flujos regionales y flujos locales, además de importantes flujos peatonales y de ciclistas. Otros segmentos con exceso de choques importante se presentan alrededor del Tunel Zurquí, en la sección más montañosa de la ruta, y en San Juan de Tibás, cerca de la capital.

Como puede notarse en la Tabla 4, aun cuando las clasificaciones por total y número equivalente de choques difieren, 15 de los 20 primeros segmentos por total de choques también se encuentran entre los 20 primeros por choques equivalentes a daños materiales. Estos 15 tramos fueron seleccionados para un análisis de ingeniería detallado y se muestran en la Figura 4.

Los tramos de concentración de choques de la Ruta 32 se localizan de manera general alrededor de los principales poblados y ciudades, como es el caso de Limón, Guápiles, Batán, Matina, Pocora y Siquirres. Otros cuatro segmentos se localizan en la parte montañosa de la carretera en los alrededores del Parque Nacional Braulio Carrillo y una última sección en los alrededores de San Juan de Tibás.

\subsection{Medidas de mitigación propuestas}

Una vez que se identificaron los tramos de concentración de choques de la Ruta 32, se procedió a realizar un análisis detallado de seguridad vial para cada uno de los sitios candidatos con base en estudios de campo e información de los choques. A continuación se detallan de manera general, tanto los principales problemas encontrados como las soluciones propuestas.

Entre los principales problemas encontrados se pueden citar la falta de anchos de carril y espaldón adecuados, señalización vertical y horizontal deficiente, baja iluminación y una gestión de los accesos inadecuada, el cual se considera como el problema principal.
Un conductor que no esté familiarizado con la carretera o esté distraído, puede generar situaciones imprevistas al encontrarse de manera inesperada el acceso en el que debe de salir. Por lo tanto, es imprescindible la colocación de mayor señalización vertical que pueda orientar a los conductores acerca de los accesos que están adelante. También se recomienda la construcción de calles marginales o travesías urbanas al paso de la Ruta 32 por los principales poblados de la zona, entre ellos se puede mencionar Guápiles, Siquirres y Limón.

La colocación de aceras apropiadas es importante para así garantizar la seguridad de los peatones que transitan al margen de la vía. Estas aceras deben estar delimitadas por una cuneta para separar de manera física los flujos peatonales de los vehiculares. Además, se propone demarcar correctamente zonas de paso peatonal, incluyendo semáforos para ese fin.

Otra de las medidas de mitigación que se plantean es implementar bahías para autobuses a lo largo del corredor, pero sobre todo en los tramos de concentración de choques aledaños a poblados importantes, pues es ahí donde se concentran las mayores poblaciones de usuarios del servicio de transporte público.

Enintersecciones importantes existenproblemas cuando se va a realizar un giro a la izquierda, pues la mayoría no cuenta con carril exclusivo para dicha maniobra, obstaculizando el paso de los vehículos que realizan el movimiento directo, lo cual puede generar choques debido a disminución súbita de velocidad. Además, la espera para poder realizar el giro izquierdo causa impaciencia, lo cual lleva tomar decisiones de realizar la maniobra cuando no tiene suficiente espacio o tiempo para hacerla, ocasionando el algunos casos colisiones. Es por esto que la implementación de carriles de giro exclusivo para la izquierda es necesaria para este tipo de intersecciones.

En los tramos de concentración de choques en la zona montañosa de la carretera se identificaron tres problemas primordiales:

- La mayoría de las barreras que existen en estos tramos no están ya en condiciones de poder seguir desempeñándose correctamente, debido a que han sido impactadas en varias ocasiones y no cumplen los estándares de diseño actuales. 


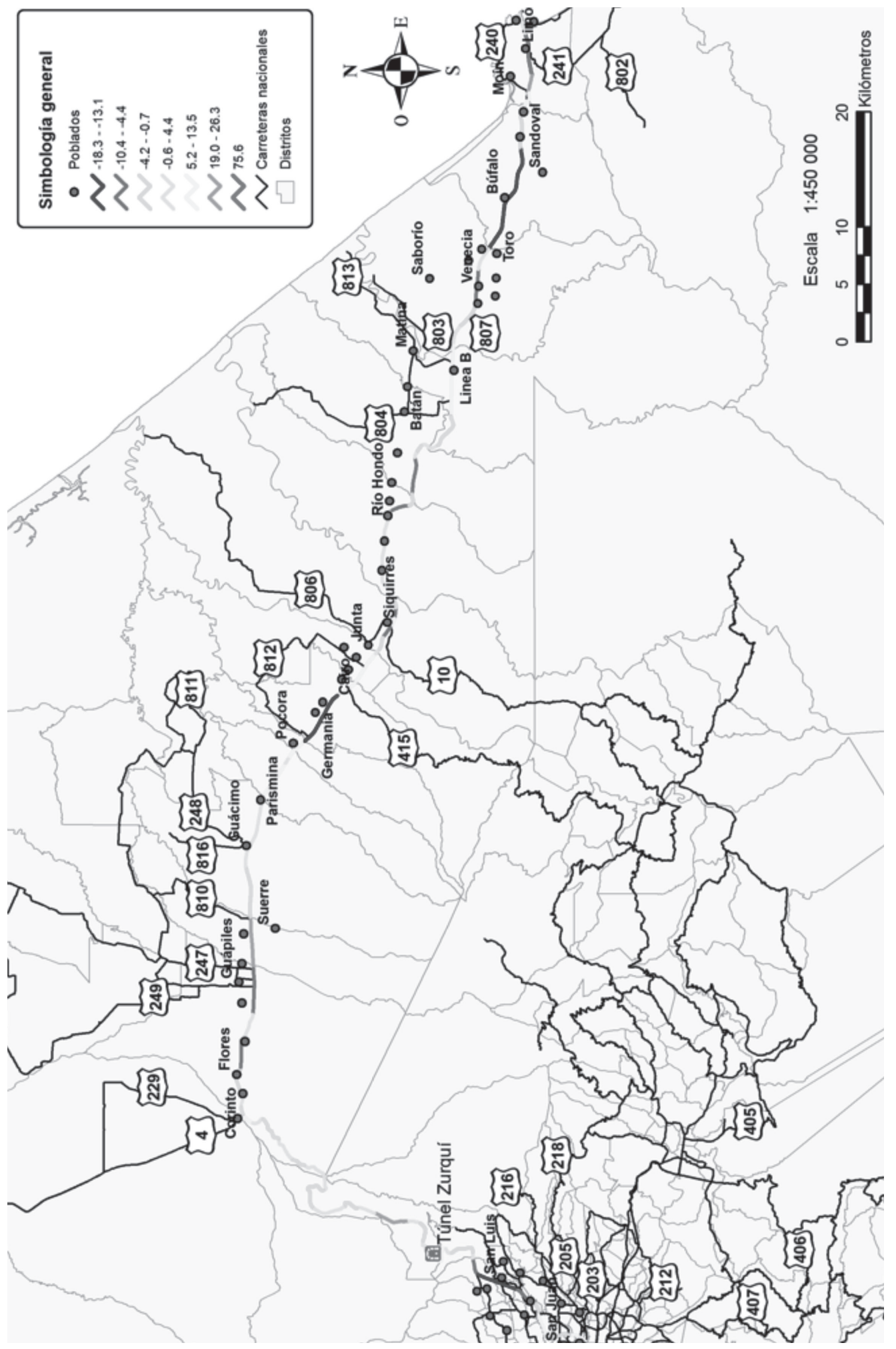

Figura 3. Mapa de exceso de choques esperado para el total de choques, Ruta 32. 
CASTRO Y AGÜERO: Aplicación del método Bayes empírico...

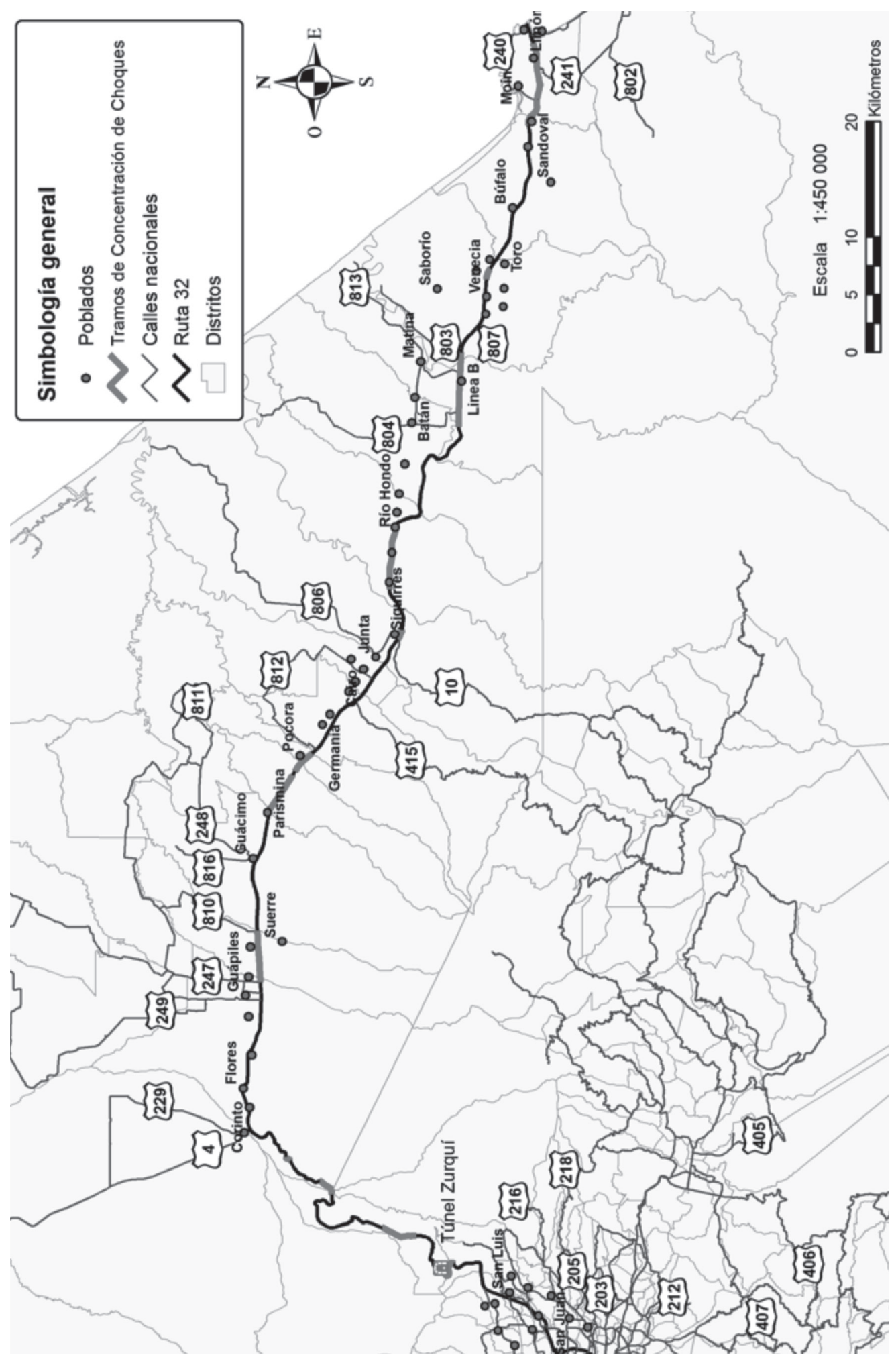

Figura 4. Mapa de tramos de concentración de choques. 
- La demarcación horizontal es prácticamente inexistente lo que complica de manera importante la tarea de manejo para los conductores, sobre todo en condiciones de clima adversas o de noche.

- La gran cantidad de transporte pesado que transita por la vía ocasiona demoras importantes, lo cual invita a conductores impacientes a realizar maniobras de rebase inseguras. Lo anterior es particularmente problemático cuando los conductores invaden el carril de acenso para realizar la maniobra de rebase en descenso, puesto que la velocidad de los pesados en descenso es también muy baja para evitar perder el control del vehículo.

Se recomienda el rediseño y reconstrucción de las barreras de seguridad en estos tramos, así como el análisis de todos los márgenes de la carretera para determinar si algunos puntos que actualmente no cuentan con barreras las necesitan. También, se recomienda una apropiada demarcación horizontal de toda la vía y la utilización de delineadores, preferiblemente verticales.

\section{CONCLUSIONES}

El método de exceso de frecuencia de choques esperado con el ajuste de Bayes empírico es apropiado para realizar un análisis de tramos de concentración de choques viales, porque es preciso, confiable y toma en cuenta tanto el sesgo de regresión a la media como el umbral de desempeño.

Se utilizó el proceso de gestión de seguridad en carreteras, incluido en el Manual de Seguridad de Carreteras, publicado por AASHTO en 2010. Este proceso también es utilizado en Europa por el Manual de Seguridad de Carretera, publicado por PIARC en 2003. El uso del mismo es apropiado para las carreteras costarricenses y conjuntamente se considera como un método actualizado y novedoso, debido a su recién publicación, pero que además es confiable para los usuarios.

El reto más complicado de este proyecto, fue la recopilación de la información de los datos de choques. Revisar archivos físicos y digitalizar todos los datos fue el proceso más largo. Es por eso que se enfatiza en la necesidad inmediata de documentar los partes de los choques viales en formato digital, para así tener esa información lista para su uso en el análisis de concentración de choques viales, lo cual sería muy beneficioso para el diseño y mantenimiento de carreteras.

Como se ha observado en experiencias de campo, y se ha mencionado a lo largo del proyecto, el espaldón en muy pocas ocasiones tiene un ancho adecuado, en caso de que exista. Los datos muestran que apenas 4 tramos de 98 tienen un espaldón mayor a 1,7 metros, que es el ancho recomendado como mínimo.

Las variables que resultaron significativas en los modelos de estimación fueron el TPDA, el número de carriles y la densidad de accesos. Se escogieron estas tres variables como base debido a que fueron las que, después de realizar varias pruebas y combinaciones de variables, resultaron ser significativas para los modelos estimados.

Los modelos de choques por categoría de gravedad no fueron estadísticamente significativos dado el bajo número de secciones en el modelo y el bajo valor esperado para estos niveles de gravedad comparado con el de total de choques. Este resultado muestra la importancia de contar no solo con los datos de choques con heridos, sino con el total de choques, de manera que los modelos estadísticos cuenten con información suficiente.

Los modelos estimados son consistentes entre sí, ya que por un lado la significancia de las variables es muy similar, y por otro lado se tiene una alta similitud en la determinación de los tramos con mayor exceso de frecuencia de choques esperado, para los modelos de total de choques así como el de equivalencia.

Contrario a lo que se pensaba al inicio del proyecto, los tramos de mayor exceso de frecuencia de choques esperado no resultaron ser los correspondientes al trayecto que cruza el Cerro Zurquí, sino más bien la mayoría (10 de 15) son después de Guápiles, en donde el alineamiento horizontal es más rectilíneo y el vertical más plano.

De acuerdo a la escogencia de tramos, la experiencia de campo, lo observado en los datos y los resultados obtenidos mediante el método de exceso de frecuencia de choques esperado con el ajuste de Bayes empírico, las medidas de mitigación de choques más relevantes para esta carretera son: 
- El ancho de carril debe tener un mínimo de 3,6 metros, incluso se podría considerar que sea más amplio debido a la gran cantidad de vehículos de carga ancha que transitan la vía. La seguridad que sienten los conductores se ve afectada cuando no tienen un ancho de carril suficiente ya que tienen menos espacio para maniobrar.

- El espaldón en casi todos los tramos es inexistente o insuficiente. En una carretera en la que la mayoría de los segmentos son de dos carriles únicamente, cualquier eventualidad que suceda - desperfectos mecánicos, llantas estalladas, choques necesita ser resuelta en el espaldón. De igual forma, en caso de que hayan peatones o ciclistas al lado de la vía, es importante que tengan suficiente espacio para desplazarse sin sentirse amenazados por los vehículos que transitan por la calzada.

- La señalización horizontal y vertical, incluyendo la delineación de la vía, es necesaria para que los conductores sean guiados de forma correcta a lo largo de la carretera. Como se indicó, la señalización vertical es muy deficiente. La demarcación, incluyendo los captadores de luz, son necesarios para guiar al usuario por la vía. Además, indicar los límites de velocidad ayudan a los mismos a conducirse mejor por la ruta.

- La colocación de aceras complementadas con cunetas en sectores en los que hay peatones es indispensable también. La seguridad de los mismos se ve afectada por la falta de aceras adecuadas para caminar o incluso esperar el transporte público. Igualmente, las aceras sirven como delimitadores e incluso ayudan a concentrar la salida y entrada de vehículos a los diferentes accesos al lado de la vía.

- Sumado a las medidas correctivas anteriores es importante destacar que, basados en los levantamientos de campo y análisis de la información disponible, se establece que la gestión de los accesos es el problema principal a atacar en la Ruta 32. En los poblados principales, se recomienda la construcción de calles marginales o travesías urbanas para reducir la frecuencia de choques.

\section{REFERENCIAS BIBLIOGRÁFICAS}

AASHTO. (2010). Highway Safety Manual. American Association of State and Highway Transportation Officials, Washington D.C. USA. Aguero-Valverde, J. (2005). Spatial Models of County-Level Roadway Crashes for Pennsylvania. MS Thesis. The Pennsylvania State University.

Aguero-Valverde, J., Jovanis, P.P. (2006). Spatial analysis of fatal and injury crashes in Pennsylvania. Accident Analysis and Prevention 38 (3), 618-625.

Castro, E.A., (2013). Análisis de tramos de concentración de choques viales en la Ruta Nacional 32, utilizando el Método de exceso de frecuencia de choques esperado con el ajuste de Bayes empírico. Trabajo Final de Graduación, Escuela de Ingeniería Civil, Universidad de Costa Rica.

Centro Centroamericano de Población (2014), Sistema de Consulta de Bases de Datos Estadísticas-Defunciones INEC [Archivo de datos]. Disponible en censos.ccp.ucr.ac.cr

Comisión Nacional de Seguridad de Tránsito (CONASET). (2008). Tratamiento de Puntos Negros con Medidas Correctivas de Bajo Costo. Gobierno de Chile, Ministerio de Transportes y Telecomunicaciones.

da Costa, T. G., Barbosa, H. M. (2014). Avaliação da Transferibilidade de Modelos de Previsão de Acidentes para Vias Urbanas em Belo Horizonte. XXVIII Congresso Nacional de Pesquisa e Ensino em Transporte da ANPET, Curitiba, Brasil. Recuperado de: http://www.anpet.org.br/xxviiianpet/anais/ documents/AC93.pdf

Department of Transport (2012). A valuation of road accidents and casualties in Great Britain in 2011. Recuperado de https://www. gov.uk/government/publications/reportedroad-casualties-great-britain-annualreport-2011.

Harwood, D W; Council, F M; Hauer, E; Hughes, W E; Vogt, A., (2000) Prediction of the expected safety performance of rural twolane highways. Midwest Research Institute; Federal Highway Administration.

Jovanis, P., Chang, H.L., 1986. Modeling the relationship of accidents to miles 
traveled. Transportation Research. Record. 1068, 42-51.

LANAMME-UCR (2011). Evaluación de la Red Vial Nacional. Identificación de zonas de riesgo de accidentes Rutas 1, 2, 32 y 34 . Programa de Infraestructura del Transporte. Laboratorio Nacional de Materiales y Modelos Estructurales. Universidad de Costa Rica. San José, Costa Rica.

World Road Association (PIARC) (2003). Road Safety Manual, Paris, Francia.

Lord, D., Washington, S. \& Ivan, J. (2005). Poisson, Poisson-gamma and zero inflated regression models of motor vehicle crashes: balancing statistical fit and theory. Accident Analysis and Prevention 37, pp. 35-46.

Miaou, S. P. (1994). The relationship between truck accidents and geometric design of road sections: Poisson versus negative binomial regressions. Accident Analysis \& Prevention, 26 (4), 471-482.

Ministry of Transport (2013). The Social Cost of Road Crashes and Injuries, 2013 Update. New Zealand. Recuperado de http://www. transport.govt.nz/research/roadcrashstatistics/ thesocialcostofroadcrashesandinjuries/socialcost-of-road-crashes-and-injuries-2013.

Oh, J., Washington, S., Lee, D. (2010). Property Damage Crash Equivalency Factors to Solve Crash Frecuncy-Severity Dilemma: Case Study on South Korean Rural Roads. Transportation Research Record 2148, 83-92.

Park, B. J., Fitzpatrick, K., \& Brewer, M. (2012). Safety Effectiveness of Super 2 Highways in Texas. Transportation Research Record: Journal of the Transportation Research Board, 2280(1), 38-50.

Peniche Mendoza, R. (2007). ¿Cuánto cuestan los Accidentes de Tránsito? Seguridad Vial, Análisis de Costos. Recuperado de http:// ebookbrowsee.net/cu\%C3\%A1nto-cuestanlos-accidentes-de-tr\%C3\%A1nsito-por-el- ing-ra\% $\% 3 \% \mathrm{BAl}$-alberto-peniche-mendozapdf-d629627618

Pennsylvania Department of Transportation (2012) 2011 Pennsylvania Crash Facts and Statistics Book. Recuperado dettp://www. dot.state.pa.us/Internet/Bureaus/pdBHSTE. nsf/InfoFb11?OpenForm.

Rizzi, L. (2001). Fotorradares y seguridad vial: un análisis empírico Bayesiano. Departamento de Ingeniería de Transporte, Pontificia Universidad Católica de Chile. Recuperado de http://www.sochitran.cl/wp-content/ uploads/Acta-2003-10-02.pdf

Shankar, V., Mannering, F., \& Barfield, W. (1995). Effect of roadway geometrics and environmental factors on rural freeway accident frequencies. Accident Analysis \& Prevention, 27(3), 371-389.

Shinar, D. (2007). Traffic Safety and Human Factor. Emerald Group Publishing Limited, UK.

World Health Organization (2013). Global status report on road safety 2013: supporting a Decade of Action. World Health Organization.

World Road Association (PIARC) (2003). Road Safety Manual, Paris, Francia.

\section{SOBRE LOS AUTORES}

Esteban Alberto Castro Estrada. Ingeniero Residente EDIFICAR S.A.

Correo electrónico: esteban.castro@outlook.com / ecastro@edificaronline.com

Tel.: 8810-0265 / 2226-2210

Jonathan Agüero Valverde. Ph.D.. Profesor Programa de Investigación en Desarrollo Urbano Sostenible Universidad de Costa Rica.

Barrio Los Profesores, Calle B, No 11, Mercedes San Pedro, San José, 11503, Costa Rica.

Correo electrónico: Jonathan.aguero@ucr.ac.cr Telefono: 506-22834927

Fax: 506-22834815 